\title{
ON MAXIMIZING AN INTEGRAL WITH A SIDE CONDITION
}

R. C. EMERSON

The problem to be discussed in this paper is that of finding an admissible function, $\hat{p}(x)$, which makes the integral

$$
\mathcal{F}(p)=\int_{\boldsymbol{E}} F(p(x), x) d x
$$

an absolute maximum subject to a side condition of the type

$$
G(p)=\int_{E} G(p(x), x) d x=c .
$$

The class, $P$, of admissible functions is to include all $p(x)$ satisfying $\mathrm{A}_{1}: u(x) \leqq p(x) \leqq v(x)$,

$\mathrm{A}_{2}: F(p(x), x)$ and $G(p(x), x)$ summable over $E$.

It will further be assumed that

$\mathrm{H}_{1}: E$ is a compact subset of the reals,

$\mathrm{H}_{2}: u(x)$ and $v(x)$ continuous for $x \in E$,

$\mathrm{H}_{3}: F(p, x)$ and $G(p, x)$ continuous for $x \in E$ and $u(x) \leqq p \leqq v(x)$,

$\mathrm{H}_{4}: \inf _{p \in \mathcal{P}} G(p)<c<\sup _{p \in \mathcal{P}} G(p)$.

Under these conditions the problem can often be solved by forming

$$
h(\theta, x)=\operatorname{Max}_{u(x) \leqq \mu \leqq 0(x)} h(\theta, x, \mu)
$$

where

$$
h(\theta, x, \mu)=\cos \theta F(\mu, x)+\sin \theta G(\mu, x) .
$$

If $\theta=\theta_{c}$ in the open interval $(-\pi / 2, \pi / 2)$ and an admissible function $\mu_{\theta_{c}}(x)$ can be found such that $\mu_{\theta_{c}}(x)$ maximizes $h\left(\theta_{c}, x, \mu\right)$ for each $x$, and if

$$
G\left(\mu_{\theta c}\right)=c,
$$

this function, $\mu_{\theta_{c}}(x)$, will be a solution to the problem. This follows because for all $x \in E$ and admissible $p(x)$

$$
h\left(\theta_{c}, x, \mu_{\theta_{c}}(x)\right) \geqq h\left(\theta_{c}, x, p(x)\right) .
$$

On integrating,

$$
\cos \theta_{c} \mathcal{F}\left(\mu_{\theta_{c}}\right)+\sin \theta_{o} G\left(\mu_{\theta_{c}}\right) \geqq \cos \theta_{c} \mathcal{F}(p)+\sin \theta_{c} G(p)
$$

Received by the editors March 9, 1953 and, in revised form, July 13, 1953. 
so that for all $p(x)$ satisfying the side condition (2)

$$
\mathcal{F}\left(\mu_{\theta_{c}}\right) \geqq \mathcal{F}(p) \text {. }
$$

Thus, the problem is solved if the existence of $\theta_{c}$ can be demonstrated. Assurance of this is given by the following theorem, and in the course of its proof an admissible $\mu_{\theta_{c}}(x)$ is constructed:

THEOREM. If $\mathcal{F}(p)$ and $G(p)$ are defined as in (1) and (2), if the class $P$ is defined by $\mathrm{A}_{1}$ and $\mathrm{A}_{2}$, and if hypotheses $\mathrm{H}_{1}, \mathrm{H}_{2}, \mathrm{H}_{3}$, and $\mathrm{H}_{4}$ are all satisfied, then there exists $\theta_{c}$ in the open interval $(-\pi / 2, \pi / 2)$ and $\mu_{\theta_{c}}(x)$ $\in \mathcal{P}$ such that for all $x \in E$

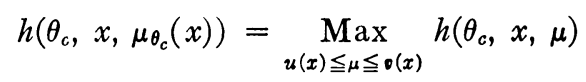

and

$$
G\left(\mu_{\theta_{c}}\right)=c,
$$

and, for all $p \in P$ such that $G(p)=c$,

$$
\mathcal{F}\left(\mu_{\theta_{c}}\right) \geqq \mathcal{F}(p) .
$$

The final assertion (11) has already been proved. In connection with proving the existence of $\theta_{c}$ we first establish the continuity of $h(\theta, x)$.

Define $F(p, x)=F(v(x), x)$ for $p>v(x)$ and $F(p, x)=F(u(x), x)$ for $p<u(x)$, similarly for $G(p, x) . h(\theta, x)$ is then the maximum of $h(\theta, x, \mu)$ over the closed interval $\inf _{x \in E} u(x) \leqq \mu \leqq \sup _{x \in E} v(x)$. As the upper envelope of the continuous functions $h(\theta, x, \mu), h(\theta, x)$ is lower semicontinuous. To show that $h(\theta, x)$ is also upper semi-continuous consider the set $(\theta, x, \mu)$ for which $h(\theta, x, \mu) \geqq \rho$. This set is bounded and closed. Its projection onto the $\theta, x$ domain is therefore also closed. This projection is, however, the set of points $(\theta, x)$ for which $h(\theta, x)$ $\geqq \rho$. Consequently, $h(\theta, x)$ is also upper semi-continuous.

We now consider for each fixed $\theta$ and $x$ the point set $\left\{\mu_{\theta}^{\alpha}(x)\right\}$ of maximizing $\mu$ 's. This set is closed by virtue of the continuity of $h(\theta, x, \mu)$ in $\mu$, and bounded by $u(x)$ and $v(x)$. Therefore, there exist functions $\mu_{\theta}^{+}(x)$ and $\mu_{\theta}^{-}(x)$ in $\left\{\mu_{\theta}^{\alpha}(x)\right\}$ satisfying

$$
\begin{aligned}
& G\left(\mu_{\theta}^{+}(x), x\right)=\sup _{\alpha} G\left(\mu_{\theta}^{\alpha}(x), x\right), \\
& G\left(\mu_{\theta}^{-}(x), x\right)=\inf _{\alpha} G\left(\mu_{\theta}^{\alpha}(x), x\right) .
\end{aligned}
$$

We shall now show that $G\left(\mu_{\theta}^{+}(x), x\right)$ is upper semi-continuous and $G\left(\mu_{\theta}-(x), x\right)$ is lower semi-continuous. Let $\left\{\theta_{j}, x_{j}\right\}$ be any sequence 
of points in the $\theta, x$ domain tending to $\left(\theta_{0}, x_{0}\right)$ for which $\mu_{\theta_{j}}^{+}\left(x_{j}\right)$ converges. Then,

$$
\begin{aligned}
h\left(\theta_{0}, x_{0}, \lim _{j \rightarrow \infty} \mu_{\theta_{j}}^{+}\left(x_{j}\right)\right) & =\lim _{j \rightarrow \infty} h\left(\theta_{j}, x_{j}, \mu_{\theta_{j}}^{+}\left(x_{j}\right)\right) \\
& =\lim _{j \rightarrow \infty} h\left(\theta_{j}, x_{j}\right) \\
& =h\left(\theta_{0}, x_{0}\right)
\end{aligned}
$$

so that

$$
\lim _{j \rightarrow \infty} \underset{\mu_{\theta_{j}}^{+}}{+}\left(x_{j}\right)=\stackrel{\alpha}{\mu_{\theta_{0}}\left(x_{0}\right)}
$$

for some $\alpha$. Consequently,

$$
\begin{aligned}
\lim _{j \rightarrow \infty} G\left(\mu_{\theta}^{+} \cdot\left(x_{j}\right), x_{j}\right) & =G\left(\lim _{j \rightarrow \infty} \underset{\mu_{\theta_{j}}^{+}}{+}\left(x_{j}\right), x_{0}\right) \\
& =G\left(\mu_{\theta_{0}}^{\alpha}\left(x_{0}\right), x_{0}\right) \\
& \leqq G\left(\mu_{\theta_{0}}^{+}\left(x_{0}\right), x_{0}\right) .
\end{aligned}
$$

Similarly, $G\left(\mu_{\theta}^{-}(x), x\right)$ is lower semi-continuous.

These functions are therefore summable over $E$, and since $F\left(\mu_{\theta}^{+}(x), x\right)$ and $F\left(\mu_{\theta}^{-}(x), x\right)$ can be expressed in terms of the $G$ 's together with $h(\theta, x)$, they too are summable over $E$. Thus $\mu_{\theta}^{+}$and $\mu_{\theta}^{-}$ are admissible functions.

Furthermore, the integral $G\left(\mu_{\theta}^{+}\right)$is upper semi-continuous and the integral $G\left(\mu_{\theta}^{-}\right)$is lower semi-continuous. This follows via Fatou's lemma, viz.

$$
\begin{aligned}
\limsup _{\theta \rightarrow \theta_{0}} \int_{E} G\left(\mu_{\theta}^{+}(x), x\right) d x & \leqq \int_{E} \limsup \sup _{\theta \rightarrow \theta_{0}} G\left(\mu_{\theta}^{+}(x), x\right) d x \\
& \leqq \int_{E} G\left(\mu_{\theta_{0}}^{+}(x), x\right) d x .
\end{aligned}
$$

Similarly, $G\left(\mu_{\bar{\theta}}^{-}\right)$is lower semi-continuous.

We now define the sets $S^{+}$and $S^{-}$as follows:

$$
\begin{aligned}
& S^{+}=\left\{\theta \mid-\pi / 2<\theta<\pi / 2 \text { and } G\left(\mu_{\theta}^{+}\right) \geqq c\right\}, \\
& S^{-}=\left\{\theta \mid-\pi / 2<\theta<\pi / 2 \text { and } G\left(\mu_{\theta}^{-}\right) \leqq c\right\} .
\end{aligned}
$$

By virtue of the semi-continuity of the integrals these sets are both closed relative to the open interval $(-\pi / 2, \pi / 2)$. Neither exhausts $(-\pi / 2, \pi / 2)$. This follows since for all $p \in \mathcal{P}$ 


$$
G\left(\mu_{-\pi / 2}^{+}\right) \leqq G(p)
$$

so that

$$
G\left(\mu_{-x / 2}^{+}\right)<c
$$

But

$$
\limsup _{\theta \rightarrow-\pi / 2} G\left(\mu_{\theta}^{+}\right) \leqq G\left(\mu_{-\pi / 2}^{+}\right)<c
$$

so that there exists $\theta \in(-\pi / 2, \pi / 2)$ such that $G\left(\mu_{\theta}^{+}\right)<c$. Thus $S^{+}$ is not equal to $(-\pi / 2, \pi / 2)$. Similarly $S^{-}$is not equal to $(-\pi / 2, \pi / 2)$.

Since $(-\pi / 2, \pi / 2)$ is connected, either

$$
S^{+} \cap S^{-} \neq 0
$$

or

$$
\operatorname{comp} S^{+} \cap \operatorname{comp} S^{-} \neq 0 \text {, }
$$

otherwise $S^{+}$and $S^{-}$would form a separation. But since $G\left(\mu_{\theta}^{+}\right)$ $\geqq G\left(\mu_{\theta}\right)$, the second alternative is absurd. We therefore pick $\theta=\theta_{c}$ in $S^{+} \cap S^{-}$. Letting

$$
\mu_{\lambda}(x)= \begin{cases}\dot{\mu_{\theta_{c}}}(x) & \text { for } x \leqq \lambda, \\ \mu_{\theta_{c}}(x) & \text { for } x>\lambda,\end{cases}
$$

we consider the integral

$$
G\left(\mu_{\lambda}\right)=\int_{x \leqq \lambda, x \in E} G\left(\mu_{\theta_{c}}^{+}(x), x\right) d x+\int_{x>\lambda, x \in E} G\left(\overline{\mu_{\theta_{c}}}(x), x\right) d x .
$$

This integral is a continuous function of $\lambda$ and covers the range

$$
G\left(\mu_{\theta_{c}}\right) \leqq c \leqq G\left(\mu_{\theta_{c}}^{+}\right) .
$$

Thus, $\lambda_{c}$ exists for which $G\left(\mu_{\lambda_{c}}\right)=c$. Therefore, $\mu_{\lambda_{c}}(x)=\mu_{\theta_{c}}(x)$ is a solution, and the theorem is proved.

The theorem above provides the basis for a maximization procedure which may be stated as follows:

In order to determine a maximizing function $\hat{p} \in \mathcal{P}$ for the integral (1) with side condition (2), $\mathrm{H}_{1}, \mathrm{H}_{2}, \mathrm{H}_{3}$, and $\mathrm{H}_{4}$ being assumed, it is necessary only to maximize the associated integrand

$$
\cos \theta F(p(x), x)+\sin \theta G(p(x), x)
$$

over the admissible values of $p(x)$ for almost all $x \in E$ and choose $\theta$ so that condition (2) is satisfied with the corresponding maximizing $p$.

Through establishing the existence of $\theta_{c}$ the theorem asserts that 
the above procedure will always yield a solution. Consequently,

COROllary 1. The problem has at least one solution.

Furthermore,

Corollary 2. Every solution $p^{*}$ conforms to the maximizing procedure-and with common $\theta$.

Assuming the denial, we put $\theta=\theta_{c}$ and have $\cos \theta_{c} F\left(p^{*}(x), x\right)+\sin \theta_{c} G\left(p^{*}(x), x\right)$

$$
<\cos \theta_{c} F\left(\mu_{\theta_{c}}(x), x\right)+\sin \theta_{c} G\left(\mu_{\theta_{c}}(x), x\right)
$$

on some subset of $E$ of measure $>0$. An integration leads to the contradiction.

Hypothesis $\mathrm{H}_{2}$ may be somewhat revised, as indicated by

Corollary 3. Bound free problems (i.e., $u(x)=-\infty$ and $v(x)$ $=+\infty)$, as well as problems with discontinuous bound functions, conform to the maximization procedure provided it is known that a bounded solution exists.

CoROllary 4. If the bound free problem has a solution $p^{\prime}$, then $p^{\prime}$ conforms to the maximization procedure on any compact subset $E^{\prime} \subseteq E$ on which $p^{\prime}$ is bounded.

Remarks. Regarding the admissibility conditions for the class $P$, the first, $A_{1}$, was imposed in order to bring problems demanding such bounds on $p$ into the domain of the maximizing principle. It may be relaxed under certain circumstances depending on the form of $F$ and $G$ (for example, see Corollaries 3 and 4 ). $\mathrm{A}_{2}$ is obviously needed. A third, $A_{3}$, imposing measurability on all $p \in P$ might possibly be included if a suitable rule could be set down (such as taking sup or inf perhaps) for selecting the $\mu_{\theta}^{+}(x)$ and $\mu_{\theta}-(x)$ at each point.

Although hypotheses $\mathrm{H}_{1}$ and $\mathrm{H}_{3}$ are about as weak as they need be for many practical problems, they might still be further weakened. This has not been investigated. On the other hand, $\mathrm{H}_{4}$ is necessary, for, consider the case $c=\operatorname{Max} G(p)$. Here a permissible $\theta$ is $\pi / 2$, and the maximizing procedure yields all $p \in \mathcal{P}$ satisfying $G(p)=c$. Some of these may yield $\mathcal{F}(p)$ larger than others.

Acknowledgments. The author wishes to thank $\mathrm{R}$. Davies and R. E. Kalaba for their critical interest in this work, and especially J. M. Danskin and M. R. Hestenes for their many helpful suggestions. 